\title{
Willingness to pay for genetic testing for inherited retinal disease
}

\author{
Sandy Tubeuf ${ }^{\star, 1}$, Thomas A Willis ${ }^{1}$, Barbara Potrata ${ }^{1}$, Hilary Grant ${ }^{1}$, Matthew J Allsop ${ }^{1}$, Mushtaq Ahmed ${ }^{2}$, \\ Jenny Hewison ${ }^{1}$ and Martin McKibbin ${ }^{3}$
}

This paper investigates the willingness of adults with inherited retinal disease to undergo and pay for diagnostic genetic testing in three hypothetical scenarios and to explore the factors that influence decision making. Fifty patients were presented with three scenarios whereby genetic testing provided increasing information: confirming the diagnosis and inheritance pattern alone, providing additional information on future visual function, and identifying in addition a new treatment which could stabilise their condition. Willingness to pay (WTP) was elicited using an iterative bidding game. Regression analysis was used to investigate the probability of agreeing to and paying for testing. Qualitative data were also reviewed to provide a comprehensive understanding of WTP and decision making. The majority of participants agreed to undergo genetic testing in each of the three scenarios. Scenario 2 was the least acceptable with $78 \%$ of participants agreeing to genetic testing. The probability of agreeing to genetic testing decreased with age. Between 72 and $96 \%$ of participants reported a WTP for genetic testing. Average WTP was $£ 539, £ 1516$, and $£ 6895$ for scenarios 1,2 , and 3 respectively. Older participants and participants with higher incomes were willing to pay more for testing. Qualitative data provided additional detail about the rationale behind participants' decisions. The study suggests that patients with inherited retinal disease were willing to undergo and to pay for diagnostic genetic testing, suggesting that they valued the information it may provide. However, several patients preferred not to receive prognostic information and were less willing to pay for genetic testing that yielded such detail.

European Journal of Human Genetics (2015) 23, 285-291; doi:10.1038/ejhg.2014.111; published online 11 June 2014

\section{INTRODUCTION}

Diagnostic testing is a growing area of interest in health-care decision making. Diagnostic tests are often evaluated according to how the results will change clinical management; however, patients may value information even if management does not change. In particular, the results of diagnostic genetic testing can provide information that is relevant to individuals and their families.

The increased availability of such expensive tests raises the question of who will pay for them. For policy makers it is not always clear whether the benefits of the tests justify the cost, and therefore it may be argued that patients could be required to pay for or contribute towards the tests themselves. One way to evaluate the monetary value of both the health and/or nonhealth benefits of genetic testing is to conduct a contingent valuation $(\mathrm{CV})$. The $\mathrm{CV}$ method consists of asking individuals directly, within a hypothetical scenario, the maximum amount that they would be willing to pay for a specific intervention or information. The willingness-to-pay (WTP) surveys have been increasingly used to evaluate health-care programmes in health and health care, ${ }^{1,2}$ including applications to predictive and diagnostic genetic testing. ${ }^{3,4}$

Recent studies in WTP have increasingly used discrete choice experiments (DCEs) to estimate WTP. ${ }^{5}$ However, we did not consider designing a DCE for the present study as it is not based on making choices between two or more discrete alternatives of genetic testing but describe the monetary value to genetic testing as a homogeneous and single-attribute good. Furthermore, DCE tasks take usually longer than CV tasks and the WTP component was not the primary focus of the interview but the last set of data collection; it required then to be a quick but reliable way of collecting the data.

For adults with inherited retinal disease, genetic testing can provide or change a clinical diagnosis, confirm the pattern of inheritance and the risk to future generations, and provide a more accurate guide to visual function. ${ }^{6}$ Although a number of centres in the United Kingdom provide both single-gene and next-generation sequencing for inherited retinal disease, access to diagnostic genetic testing within the publicly funded National Health Service (NHS) remains variable. ${ }^{7}$ Few studies have explored patient attitudes towards genetic testing for inherited retinal disease. The available evidence suggests that patients are generally enthusiastic towards testing and feel that it should be available, even if they may choose not to utilise it themselves. ${ }^{8-11}$ Recently, Eden et $a l^{4}$ examined the monetary value that individuals with and without prior experience of retinitis pigmentosa placed upon genetic counselling and testing. Responding to a hypothetical scenario, the majority of their sample reported that they would seek genetic counselling and testing.

In this study, a CV method was used to investigate the selfperceived value of diagnostic genetic testing in various scenarios among adults with inherited retinal disease. The use of a WTP survey permits the examination of the relative value of differing outcomes of testing. We explored how different variables (eg, age, income) may be

${ }^{1}$ Leeds Institute of Health Sciences, University of Leeds, Leeds, UK; ${ }^{2}$ Yorkshire Regional Genetics Service, Chapel Allerton Hospital, Leeds, UK; ${ }^{3}$ Department of Ophthalmology, St James's University Hospital, Leeds, UK

${ }^{*}$ Correspondence: Dr S Tubeuf, Academic Unit of Health Economics, Leeds Institute of Health Sciences, University of Leeds, Charles Thackrah Building, 101 Clarendon Road, Leeds LS2 9LJ, UK. Tel: +44 (0)113 343 0843; Fax: +44 (0)113 343 3470; E-mail: s.tubeuf@leeds.ac.uk

Received 10 December 2013; revised 13 March 2014; accepted 8 May 2014; published online 11 June 2014 
associated with decisions to undergo genetic testing and the magnitude of the WTP value. Moreover, qualitative data were collected alongside the WTP values, allowing consideration of the reasoning behind individual participant responses.

\section{MATERIALS AND METHODS \\ Participants}

The sample comprised 50 adults with a range of inherited retinal diseases who were selected for in-depth interviews from a larger sample of 200 research participants. The details of recruitment and the main study design are reported elsewhere; ${ }^{10}$ it included participants having been diagnosed with a range of inherited retinal dystrophies, both congenital and acquired, and generalised and localised to the macula. Selection of these 50 people was based on a purposive sampling frame, representing a maximal diversity of self-reported levels of knowledge and demographic characteristics. Eligibility criteria for the original study included: age over 16 years and a clinical diagnosis of inherited retinal disease but no significant hearing impairment (to facilitate telephone interviews). Participants were selected for this subsequent face-to-face interview on the basis of their demographic characteristics and other factors such as attitudes towards their condition, reported in the original study. Ethical approval was received from the Leeds (East) Research Ethics Committee $(10 / \mathrm{H} 1306 / 90)$ and informed consent was obtained from all participants. The full questionnaire of the in-depth interview is available upon request to the authors.

\section{Survey design}

Participants were presented with three hypothetical scenarios whereby genetic testing provided increasing information relevant to the inherited retinal disease. They were asked to imagine that the NHS would not pay for diagnostic genetic testing but that they could pay to have the test done in an NHS laboratory. The hypothetical scenarios are presented in Figure 1. The level of information provided by the testing increased additively across the three scenarios: in scenario 1, patients were told that the test would 'confirm (or possibly alter) their diagnosis and how the condition is inherited, helping to explain the risk to other generations'; in scenario 2, the test would do this and give 'a more accurate idea of your eyesight in the future'; in addition to this information, scenario 3 would also 'identify a new treatment that could stabilise your eye condition'. It was emphasised that the test described in scenario 3 was not currently available, but participants were required to respond as if it were. All patients received the same three scenarios presented in the same order. The level of risk did not vary; participants were told that the test was $100 \%$ accurate in each case. The scenarios were designed by the research team, approved by the study Steering Committee, and tested in three pilot interviews before use. The rationale behind the three scenarios was to begin with two outcomes that might reasonably be offered at a contemporary clinic, whereas the third scenario was accepted as currently being very much hypothetical. It was not considered important to include in addition a level of

\begin{tabular}{|l|l|}
\hline Scenario 1 & $\begin{array}{l}\text { A genetic test is available that will confirm (or possibly alter) the } \\
\text { diagnosis of your retinal disease, and how the condition is } \\
\text { inherited, helping to explain the risk to other generations. } \\
\text { The test is 100\% accurate and you will have to pay the whole cost. }\end{array}$ \\
\hline Scenario 2 & $\begin{array}{l}\text { As before, a genetic test is available that will confirm or possibly } \\
\text { alter the diagnosis, tell you how the condition is inherited and will } \\
\text { also give you a more accurate idea of your eyesight in the future. } \\
\text { Again, the test is 100\% accurate but you will have to pay the cost. }\end{array}$ \\
\hline Scenario 3 & $\begin{array}{l}\text { Scenario } 3 \text { is more hypothetical. Again, a genetic test is available } \\
\text { that will confirm the diagnosis, tell you how the condition is } \\
\text { inherited and will give you a more accurate idea of your eyesight } \\
\text { in the future. This time it will also identify a new treatment that } \\
\text { could stabilise your condition. This test is not available at the } \\
\text { present time but we are interested in your response as if it were. } \\
\text { Again, the test is 100\% accurate and you will have to pay the cost. }\end{array}$ \\
\hline
\end{tabular}

Figure 1 The willingness-to-pay question in each scenario. uncertainty into the outcome as our main aim was to get an idea of how much people valued different outcomes.

The maximum WTP was elicited using an iterative double-bounded, binary bidding game (yes/no) having the same predetermined pattern across scenarios and for all participants. The initial bidding was $£ 250$ and participants were asked if they would pay this amount. Respondents answering 'yes' to the starting bidding were asked if they would pay twice the initial price. Respondents answering 'no' were asked if they would pay half the initial price. If the second response was also 'no', respondents were asked if they would be willing to take the test for free. The survey elicited the respondent's upper boundary on their WTP as any respondent answering 'yes' to increasing bidding or 'yes' to undergoing testing for free were ultimately asked the maximum amount of money they would pay for the test. All monetary values are reported in UK pounds sterling.

Both quantitative and qualitative data were collected on the willingness to undergo testing and the elicited WTP for the test in each scenario. Each scenario was completed with prompts or further questions for participants to explain and comment on their level of WTP. Sociodemographic information was collected in the initial study; self-reported annual income was collected as part of this study. The WTP questionnaire took $\sim 10 \mathrm{~min}$ to complete. Answers were audio-recorded and transcribed verbatim.

\section{Analysis}

A descriptive analysis of the willingness to undergo and pay for genetic testing across scenarios was undertaken. A two-part model was implemented when at least some participants declared a nonpositive WTP and a single-part model was used otherwise. First, the probability of agreeing to genetic testing was estimated, including individuals who would undergo the test only if it was free; probit regression models were used to test factors associated with individuals' decision to take the test. Second, analyses focussed only upon those individuals who were willing to pay for the test (WTP $>0$ ) and explored the association of individual characteristics with the log-amount that individuals reported that they would pay for the test. The log transformation of the WTP amount was used to allow the use of ordinary least square modelling. Both models included the same set of predicting factors: age in years, gender, occupation status (categorised as professional, manual/clerical post, unemployed/inactive), having or wishing to have children, annual income category (more or less than $£ 20000$ a year), and self-reported level of understanding of what a genetic test involves on a 0 (poor) to 5 (high) scale dichotomised as 0-3 versus 4-5.

Quantitative results were combined with qualitative analysis to interpret the findings. Qualitative data were coded independently and subsequently analysed by two researchers (BP and $\mathrm{HG}$ ). Coding results were compared and differences resolved by consensus. The statements were analysed using a thematic approach, a common analytical method in this area ${ }^{12,13}$ and managed using NVivo 8 (QSR International Pty Ltd., Version 8, Burlington, MA, USA).

\section{RESULTS}

\section{Test uptake and WTP}

Descriptive statistics of the sample are provided in Table 1. The sample comprised equal numbers of males and females, with a mean age of 46.7 years (SD 15.9, range 18-74). Table 2 reports the proportion of all respondents who would undergo diagnostic testing in each of the three scenarios, the proportion that would pay for testing, and the average WTP values.

The majority of participants were willing to undergo testing across the three scenarios (ie, they would pay or have it for free). Testing in scenario 3 would be taken up by $100 \%$ of the respondents, whereas testing in scenario 2 was the least attractive, with only $78 \%$ of the respondents agreeing to testing. The figures were slightly reduced when considering only those who would be willing to pay for genetic testing. Scenario 3 was again the most attractive, with $96 \%$ of participants expressing a WTP $>£ 0$ for testing, and scenario 2 was the least attractive, with only $72 \%$ reporting a WTP $>£ 0$ for testing. 
Table 1 Sample statistics

\begin{tabular}{|c|c|}
\hline Variables & Mean (SD) \\
\hline \multirow[t]{2}{*}{ Age in years } & 46.7 (15.9) \\
\hline & Frequency (\%) \\
\hline Female & $25(50 \%)$ \\
\hline \multicolumn{2}{|l|}{ Religion } \\
\hline Christian, Muslim, Buddhist & $27(54 \%)$ \\
\hline No religion & $23(46 \%)$ \\
\hline \multicolumn{2}{|l|}{ Occupation status } \\
\hline Professional & $18(36 \%)$ \\
\hline Manual, clerical staff & $6(12 \%)$ \\
\hline Not working, unemployed & $26(52 \%)$ \\
\hline \multicolumn{2}{|l|}{ Children } \\
\hline Have or wish to have children & $38(76 \%)$ \\
\hline No plans to have children & $12(24 \%)$ \\
\hline \multicolumn{2}{|l|}{ Self-reported annual income } \\
\hline$>£ 20000$ & $25(50 \%)$ \\
\hline$<£ 20000$ & $25(50 \%)$ \\
\hline \multicolumn{2}{|l|}{ Extent of understanding genetic test ( $0-5$ high) } \\
\hline $0-3$ & $29(58 \%)$ \\
\hline $4-5$ & $21(42 \%)$ \\
\hline \multicolumn{2}{|l|}{ Diagnosis } \\
\hline Retinitis pigmentosa & $21(42 \%)$ \\
\hline Macular dystrophy & $5(10 \%)$ \\
\hline Stargardt disease & $7(14 \%)$ \\
\hline X-linked retinoschisis & $2(4 \%)$ \\
\hline Best disease & $4(8 \%)$ \\
\hline Choroideremia & $1(2 \%)$ \\
\hline Sorsbys & $3(6 \%)$ \\
\hline Retinal dystrophy & $2(4 \%)$ \\
\hline Enhanced S-cone syndrome & $1(2 \%)$ \\
\hline Central areoalar chorioretinal dystrophy & $2(4 \%)$ \\
\hline Leber congenital amaurosis & $1(2 \%)$ \\
\hline Cone dystrophy & $1(2 \%)$ \\
\hline
\end{tabular}

The number of respondents unwilling to pay any amount for testing represented $10 \%$ in scenario 1 and $>20 \%$ in scenario 2 .

Median WTP increased across the three scenarios: $£ 300$ in scenario 1 , $£ 500$ in scenario 2, and $£ 1250$ in scenario 3 . On average, respondents were willing to pay $£ 539$ for the test in scenario 1, $£ 1516$ in scenario 2, and $£ 6895$ in scenario 3. A majority of respondents (72-96\%) would be willing to pay something for testing. When we excluded patients who would only agree to testing if it were free, the average WTP increased and ranged from $£ 591$ for scenario 1 to $£ 7182$ for scenario 3 .

As displayed in Figure 2, the willingness to pay a high monetary value for genetic testing increased with the scenarios and the potential information to be provided by the test; $36 \%, 44 \%$, and $68 \%$ of patients were willing to pay more than $£ 500$ for testing in scenarios 1 , 2 , and 3, respectively. For lower WTP categories, a different pattern was observed: patients were always willing to pay more for testing in scenario 1 than in scenario 2 . There was a tendency for patients to be less inclined to agree to testing in scenario 2, but if they did agree to testing in this situation, then they were willing to pay more for testing than in scenario 1 .

Table 3 reports the multivariate regression results. There were no significant predictors for whether participants were inclined to undergo genetic testing outlined in scenario 1. Two predictors emerged for the probability of agreeing to testing in scenario 2: age and self-reported level of understanding about genetic testing. Older respondents were less likely to undergo testing, as were those who considered themselves to have a high level of understanding, all other things being equal.

Considering only the respondents who were willing to pay a positive WTP for testing, age was a significant factor predictor across all three scenarios: WTP amount increased with age, all other things being equal. Finally, the WTP value in scenario 3 was significantly associated with income: participants with higher incomes were more likely to be willing to pay more for testing.

\section{Qualitative interviews}

Qualitative data were analysed in relation to each scenario and then as response patterns. Illustrative quotes are presented in Box 1.

Scenario 1. A majority of participants (90\%) reported willingness to undergo testing, with $82 \%$ of the sample willing to pay for it.

Table 2 Willingness-to-pay (WTP) value in GBP $(£)$ by scenario

\begin{tabular}{|c|c|c|c|}
\hline & Scenario 1 & Scenario 2 & Scenario 3 \\
\hline \multicolumn{4}{|l|}{ All respondents, $n=50^{\mathrm{a}}$} \\
\hline Share of the sample agreeing to testing & $90 \%$ & $78 \%$ & $100 \%$ \\
\hline Median & $£ 300$ & $£ 500$ & $£ 1250$ \\
\hline Mean & $£ 539$ & $£ 1516$ & $£ 6895$ \\
\hline SD & $£ 793$ & $£ 4176$ & $£ 15257$ \\
\hline Min-max & $£ 0-£ 5000$ & $£ 0-£ 25000$ & $£ 0-£ 100000$ \\
\hline \multicolumn{4}{|c|}{ Only those respondents who would pay for testing $(W T P>£ O)^{\mathrm{b}}$} \\
\hline Share of the sample who would pay for testing & $82 \%$ & $72 \%$ & $96 \%$ \\
\hline Median & $£ 300$ & $£ 500$ & $£ 1500$ \\
\hline Mean & $£ 591$ & $£ 1643$ & $£ 7182$ \\
\hline SD & $£ 812$ & $£ 4326$ & $£ 15510$ \\
\hline Min-max & $£ 50-£ 5000$ & $£ 50-£ 25000$ & $£ 250-£ 100000$ \\
\hline
\end{tabular}

ancludes those who would be willing to pay for testing and those who would undergo testing if it was free.

bExcludes those who would not undergo testing or would only undergo testing if it was free. 
A common theme was that a confirmed diagnosis and details of the inheritance pattern would not be of significant personal benefit but more of use to their family members. Individual circumstances were frequently reported, particularly age and family status, as justification for their WTP response: testing was considered to be most relevant at a particular time of life. For example, several younger respondents were interested in testing but would consider it more important when they were planning families, or once existing children were able to express a desire to learn more about the condition. Several older participants meanwhile were less enthusiastic about testing, often because they were already severely sight-impaired or because their children were now adults and they believed that they had not inherited the condition.

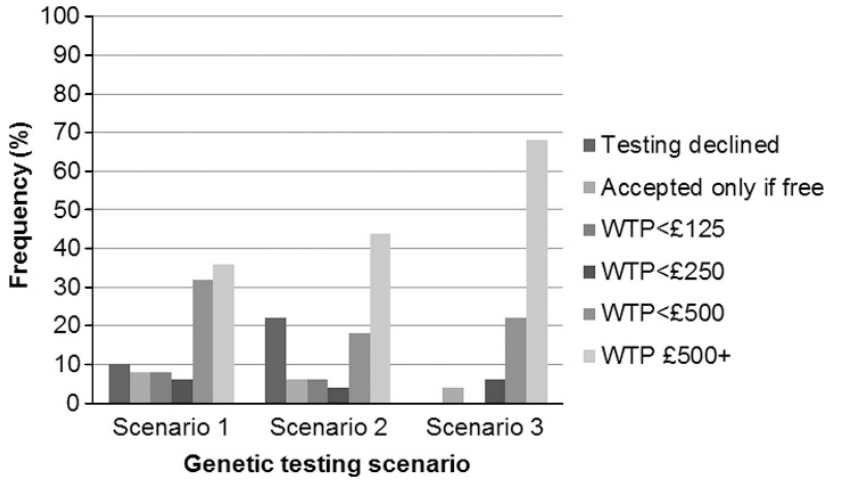

Figure 2 Willingness to undergo and pay for genetic testing by scenario.
Nine participants (18\%) reported that they would not be willing to pay for testing, although most of these would accept it if free. Among this group, a typical reason was that it would not add anything beyond what they have already been told by their consultant. Others also explained that receiving a confirmed/changed diagnosis would not actually change their vision, and thus they considered the test was of little benefit.

Scenario 2. Scenario 2 included the same information as in scenario 1, together with additional detail about future eyesight. On average, there was an increase in the amount that participants were willing to pay. Closer inspection of the data revealed some interesting differences in views and attitudes about this test scenario.

Whereas 19 participants (38\%) considered this test to have added value (ie, they were willing to pay more than they had for scenario 1 ), $22(44 \%)$ reported the same WTP value as for testing in scenario 1 , and several participants stated that they would not agree to testing, even if free. These individuals reported that they preferred not to know the future extent of their sight loss if they were unable to change it. Some described the potential emotional consequences of receiving such information, for example, feeling that this knowledge would 'hang over' them or induce worry and anxiety. Participants holding an opposing view explained that this new information would be desirable and would justify further expense. A common rationale was that it would permit future planning: if they had few years of good eyesight remaining, they might choose to spend more money now (eg, on holidays) or save more to prepare for being unable to remain in their current job. When it was put to these individuals that several others would decline this test, some described this attitude as 'putting your head in the sand'.

Table 3 Regression results

Probability of agreeing to testing

Log of the average WTP for testing

Scenario $1 n=50 \quad$ Scenario $2 n=50 \quad$ Scenario $1 n=41 \quad$ Scenario $2 n=36 \quad$ Scenario $3 n=48$

\begin{tabular}{|c|c|c|c|c|c|c|c|c|c|c|}
\hline Variables & Coefficient & $S E$ & Coefficient & $S E$ & Coefficient & $S E$ & Coefficient & $S E$ & Coefficient & $S E$ \\
\hline Age in years & -0.006 & 0.029 & $-0.056^{*}$ & 0.023 & $0.022 *$ & 0.008 & $0.040 *$ & 0.016 & $0.027^{*}$ & 0.014 \\
\hline \multicolumn{11}{|l|}{ Gender } \\
\hline Female & -0.184 & 0.626 & -0.647 & 0.579 & -0.406 & 0.253 & -0.203 & 0.432 & -0.422 & 0.413 \\
\hline Male & Ref. & & Ref. & & Ref. & & Ref. & & Ref. & \\
\hline \multicolumn{11}{|l|}{ Occupation status } \\
\hline Professional & 1.265 & 0.883 & 1.551 & 1.091 & -0.794 & 0.510 & 0.074 & 0.699 & -0.684 & 0.715 \\
\hline Manual, clerical staff & Ref. & & Ref. & & Ref. & & Ref. & & Ref. & \\
\hline Not working, unemployed & $1.564^{*}$ & 0.907 & 0.323 & 0.854 & -0.541 & 0.502 & -0.222 & 0.699 & 0.087 & 0.669 \\
\hline \multicolumn{11}{|l|}{ Children } \\
\hline Have or wish to have children & 0.913 & 0.772 & 0.852 & 0.669 & -0.069 & 0.324 & 0.015 & 0.578 & -0.303 & 0.522 \\
\hline No plans to have children & Ref. & & Ref. & & Ref. & & Ref. & & Ref. & \\
\hline \multicolumn{11}{|l|}{ Self-reported annual income } \\
\hline$>£ 20000$ & -0.843 & 0.715 & 0.708 & 0.699 & 0.503 & 0.328 & -0.422 & 0.535 & $1.504^{* *}$ & 0.502 \\
\hline$<£ 20000$ & Ref. & & Ref. & & Ref. & & Ref. & & Ref. & \\
\hline
\end{tabular}

Self-reported understanding of genetic testing ( $0-5$ high)

\begin{tabular}{|c|c|c|c|c|c|c|c|c|c|c|}
\hline $0-3$ & Ref. & & & & & & & & & \\
\hline $4-5$ & -0.056 & 0.602 & $-2.208^{* *}$ & 0.826 & -0.007 & 0.238 & 0.769 & 0.435 & 0.101 & 0.418 \\
\hline Intercept & 0.469 & 2.051 & 4.297 * & 1.874 & $5.527^{* *}$ & 0.730 & $4.965^{* *}$ & 1.517 & $6.753^{* *}$ & 1.332 \\
\hline
\end{tabular}

${ }^{*} P<0.05$ and ${ }^{* *} P<0.01$ 


\section{Box 1 Illustrative qualitative comments relating to each scenario}

\section{Scenario 1}

It's more from the child point of view, that's the important factor for me I think, how's this going to impact on my family, my children, future generations... [ID024, male, 53 years old].

'No, not now, if perhaps I was child-inclined... Again, if I was at the child bearing stage of my life I'd pay quite a lot I think. I think I'd even pay up to $£ 10,000$ ' [ID075, female, 34].

'I think that I probably would to get a clear... diagnosis [...] exactly what were wrong with me. And then you'd know if there were any treatments available or anything' [ID111, female, 21].

'...it’s almost saying do you want to pay $£ 250$ to know what you already know' [ID115, male, 46].

'well at 68 I don't think it's any use to me now because I'm registered blind so it doesn't make any difference does it?' [ID086, female, 68].

\section{Scenario 2}

'I know what my eyesight's gonna be in the future: nothing! So it would be a bit pointless really. ... I don't even know if I'd want to know if I had some sight... I'd probably go away and just worry about it all the time' [ID002, female, 61]

'it would make me depressed thinking that maybe I would lose my sight at a particular...I think l'd rather know I've got the condition and just wait and see what happens' [ID007, female, 55]

'... what we're talking about is would I want to know if it was going to stay the same or it was going to get worse, because I know it's not going to get better. It's kind of like signing your own death warrant. I don't think I'd want to know because it would be hanging over me. ... I don't know whether it's worse to not know how it's going to progress or it's worse to know how it's going to progress. I am tempted to say I think to an extent ignorance is bliss and I think I'd sooner not know' [ID151, male, 46]

'... how long I'm going to maintain useable vision, which is a pretty useful lifeplanning tool. It would be very handy to have because I want to go and blow the lot on doing a few more good holidays and things like that' [ID011, male, 68] 'I would definitely consider $£ 1-2000$ just to know if they could tell me exactly when to the nearest year...I would completely lose my sight, just so I knew and maybe build up my savings or some kind of retirement plan. Or just case of right I need to start learning...। don't even know how to use Braille at this point in time, just that kind of thing' [ID053, male, 39]

\section{Scenario 3}

'Oh crikey! Yes, definitely! Oh... how high would you go? ... you would probably pay absolutely whatever you could. Wouldn't you?' [ID013, female, 40]

'If you were offering me that today in my current situation, I would probably be saying $£ 10-15,000$, because it'd be worth an awful lot more than that to me in the long run' [ID064, male, 39]

'I'd give you my bank book. Does that answer you? ... I'd pay that money because it gives you the priceless gift of sight and you can't put a price on that' [ID114, male, 66]

'Well no, because there's nothing to stabilise. I've lost all useful sight now' [ID002, female, 61]

'No, because I already know from my consultant that my eyesight shouldn't get any worse' [ID038, female, 26]

An interesting observation from testing in scenario 2 was that participants tended to have a negative expectation about the outcome of such a test - none considered that their prognosis might produce good news about their future sight.

Scenario 3. Responses to scenario 3 (where the test would provide additional information about treatment that would stabilise the condition) were overwhelmingly positive, as demonstrated by the substantial increase in the amounts that participants stated they would be willing to pay. Typically, even those who had declined the initial two options would agree to testing in this scenario.

Many respondents found it difficult to elicit a WTP value in this scenario; several participants reported that they would pay whatever they were able. One participant made the comparison with cosmetic surgery, explaining that stabilising sight was a far more worthwhile expense. Some also reported that even if they did not have the money to pay for testing, they would be prepared to fundraise for it.

Nevertheless, a minority felt that they would not be willing to pay much for this test or in some cases not pay anything at all. These tended to be individuals whose eyesight had already declined to such a degree that they had little or no sight remaining. Hence, a treatment to stabilise their vision would be of little benefit. Similarly, some who had good vision explained that they had been informed that their vision was unlikely to deteriorate further and hence were content as they were.

Response patterns. Approximately a third of respondents $(n=16$; $32 \%$ ) demonstrated an 'additive' pattern, that is, raising the amount that they would pay with each step. The additional knowledge gained at each step was considered beneficial and justified additional expense.

The most common pattern, demonstrated by $21 \quad(42 \%)$ participants, showed scenarios 1 and 2 to be considered of equal worth (this includes those who would have both tests if free only), with an increase for scenario 3. For these individuals, a test that would offer prognostic information (scenario 2) was not considered of greater value than a test providing diagnostic and inheritance pattern information alone (scenario 1).

Three other patterns were identified, demonstrated by smaller numbers of participants. Seven respondents (14\%) valued scenario 2 lower than scenario 1 . The two remaining patterns saw scenarios 2 and 3 valued equally, as either more attractive $(n=4,8 \%)$ or less attractive $(n=2,4 \%)$ than scenario 1 . Inspection of qualitative data revealed that the two individuals who reported being willing to pay more for scenario 1 than the two other scenarios were both $>60$ years old and had severe sight loss. They perceived neither benefit from prognostic information nor from stabilising their existing level of vision.

\section{DISCUSSION}

The results of this study suggest that diagnostic genetic testing for inherited retinal disease is highly valued by patients. The increasing WTP pattern was not universal, however. Scenario 2, which included prognostic information about future visual function, was not popular with all participants and showed the lowest levels of acceptance. Indeed, $62 \%$ of the sample indicated that they would not be willing to pay more for this test than what they would pay for the test in scenario 1, suggesting that the prognostic information was not considered to be of additional benefit. Several respondents also stated that they would not be willing to pay for testing that yielded prognostic information, or even agree to it if it was free.

The results of the regression analyses revealed that some individual factors predicted both the likelihood of undergoing testing and the amount that participants would be willing to pay. Both age and selfreported understanding of genetic testing were significantly negatively associated with the probability of agreeing to testing in scenario 2 . Several older participants were not willing to undergo this test because their eyesight was already severely impaired and they reported that they did not wish to know the extent of any future deterioration.

The amount of money participants reported being willing to pay for testing was positively associated with age (all scenarios) and 
income (scenario 3 only). This finding appears unsurprising, with older participants arguably being able to access greater amounts to pay for a test if desired. It is not clear why a higher self-reported understanding of genetic testing would make acceptance of scenario 2 less likely but two tentative explanations could be offered. It may be that those participants who feel that they possess a good understanding of testing are happy with their current knowledge. Thus, although confirmation of a diagnosis and potential treatments would appeal, additional information about their future eyesight would be less valuable to them. Another possible explanation is that these people are aware of the limitations of 'more accurate' tests, that is, they realise that the prediction of their future eyesight might still be wrong.

The present study is among the first to document the monetary value that patients with inherited retinal disease would assign to diagnostic genetic testing. For example, Eden et al ${ }^{4}$ concluded that WTP was higher for genetic testing with counselling than for genetic counselling alone, with median values of $£ 524$ and £224.50, respectively. Their sample consisted of individuals affected by retinitis pigmentosa, as well as unaffected individuals. Our findings are consistent with other research in this area. For example, Graves et $\mathrm{al}^{14}$ found that greater overall interest in genetic testing for cancer was related to higher levels of cancer worry and greater perceived benefits of genetic testing.

For retinal disease, there is uncertainty over the current demand for genetic testing, ${ }^{15}$ with no clear consensus on willingness to utilise and pay for genetic testing. There have been reports of considerable public interest surrounding genetic tests that are offered direct to the consumer, even when an immediate clinical value is not identified. It is not surprising that participants in this study demonstrated a high WTP in scenario 3 as this scenario provided opportunity for clinical benefit or possible applications to decision making about one's life. Given the willingness to undergo testing and demonstrated WTP, concurrent consideration should be given to the need to develop support services that adequately mitigate for negative consequences (the burden of the knowledge, emergence of moral dilemmas around reproduction, potential impact on insurance) that could emerge from testing. $^{11}$

Research on the WTP for diagnostic technologies is timely and has been explored for various diseases. ${ }^{3,16}$ The inclusion of qualitative data permitted a deeper exploration of the issues, with responses to scenario 2 of particular interest. The majority of participants reported that they were willing to take a test that would provide more accurate information about their future eyesight. However, a sizeable minority reported that they would not be interested in such a test, regardless of whether or not they had to pay for it. Some respondents reported that the emotional consequences of receiving such knowledge would be dramatic and potentially harmful, and this was the main reason for declining the test. This study suggests that participants value genetic testing and new technologies. Therefore, it warrants the provision of accurate genetic counselling to such individuals where they are fully informed about the new advances together with its limitations and their expectations are managed. Recent work from our group has demonstrated that the information needs of many patients with inherited retinal disease appear to be unmet. ${ }^{17}$

Our study presents a number of limitations of the experimental nature of CV surveys. ${ }^{18,19}$ Typically, the reported willingness to take the test and to pay for testing in this study is only a proxy for actual uptake of testing and true willingness to pay. In addition, our survey design, particularly the wording of the questionnaire, the sequence in which scenarios were presented, and the initial bidding amount, is likely to have influenced the WTP responses. A larger study sample would facilitate the use of alternative starting bids, and we could randomly assign respondents to different starting-point groups in order to ensure homogeneity and to measure the starting bid effects. It is also important to remind that the three scenarios were fictional; we considered that the test was $100 \%$ accurate despite the fact that this is unlikely to be realistic. This choice was motivated by our focus on eliciting the relative value of the perceived benefits of the test and controls other dimensions of fear and doubt about the test. In future work, the impact of various levels of uncertainty on the WTP for genetic testing should be considered. The study sample, despite being larger than other similar studies, ${ }^{4,9}$ was relatively small and our results may lack statistical power and may not be considered representative of the wider population of those affected with inherited retinal diseases. However, when populating the sampling frame, effort was made to include individuals of varying age, gender, and ethnicity, as well as retinal condition. Retinitis pigmentosa was the most common diagnosis in the present sample (42\%), matching its position as the most common inherited retinal condition. ${ }^{20,21}$ Finally, the sample was a purposive one, drawn from a wider study; ${ }^{10}$ this means that although participants were deliberately selected to gather a range of views, further research is necessary to assess whether such views are held more widely; the use of a control group in the study would be useful for this purpose. Our participants may be more invested in obtaining genetic information than the general public, and hence our results are expected to overestimate interest in and willingness to pay for genetic testing in the context of inherited retinal disease. Finally, this study is a small experimental study, and hence the elicited WTP values can only be used in a descriptive sense and could not guide commissioners to develop actual costing of genetic tests.

This study suggests that most adults with inherited retinal disease are willing to undergo and pay for diagnostic genetic testing, even when it does not identify a therapy. The findings support other research that has identified the importance attached to genetic testing and genetic counselling support for inherited retinal disease. However, it is clear that people's view on testing are different, and hence policy makers and practitioners should not assume that the public are uniformly viewing more tests as a good thing.

\section{CONFLICT OF INTEREST}

The authors declare no conflict of interest.

\section{ACKNOWLEDGEMENTS}

This paper presents independent research funded by the National Institute for Health Research (NIHR) under its Research for Patient Benefit (RfPB) Programme (Grant Reference Number PB-PG-0909-20228). The views expressed are those of the author(s) and not necessarily those of the NHS, the NIHR or the Department of Health. We are grateful to Peter Neumann for sending a copy of the basic survey used for a study on WTP for predictive tests published in Health Economics 2012(21):238-251. We would like to thank Richard Edlin for comments on a previous version of this article.

1 Olsen JA, Smith R: Theory versus practice: a review of 'willingness-to-pay' in health and health care. Health Econ 2001; 10: 39-52.

2 Sach TS, Smith RD, Whynes DK: A 'league table' of contingent valuation results for pharmaceutical interventions: a hard pill to swallow. Pharmacoeconomics 2007; 25: 107-127

3 Neumann PJ, Cohen JT, Hammitt JK et al: Willingness-to-pay for predictive tests with no immediate treatment implications: a survey of US residents. Health Econ 2012; 21: $238-251$. 
4 Eden M, Payne K, Combs RM et al: Valuing the benefits of genetic testing for retinitis pigmentosa: a pilot application of the contingent valuation method. $\mathrm{Br} \mathrm{J}$ Ophthalmol 2013; 97: 1051-1056.

5 Ryan M: A comparison of stated preference methods for estimating monetary values. Health Econ 2004; 13: 477-492.

6 Koenekoop RK, Lopez I, den Hollander Al et al: Genetic testing for retinal dystrophies and dysfunctions: benefits, dilemmas and solutions. Clin Exp Ophthalmol 2007; 35: 473-485.

7 Moore T, Burton H: Genetic ophthalmology in focus: a needs assessment and review of specialist services for genetic eye disorders. Report for the United Kingdom Genetic Testing Network, PHG Foundation, 2008. http://www.phgfoundation.org/pages/ projectlist.htm.

8 Bong C, Potrata B, Hewison J et al: Attitudes of patients and relatives/carers towards genetic testing for inherited retinal disease. Eye 2010; 24: 1622-1625.

9 Mezer E, Babul-Hirji R, Wise $\mathrm{R}$ et al: Attitudes regarding predictive testing for retinitis pigmentosa. Ophthalmic Genet 2007; 28: 9-15.

10 Willis T, Potrata B, Ahmed $\mathrm{M}$ et al: Understanding of and attitudes to genetic testing for inherited retinal disease. Br J Ophthalmol 2013; 97: 1148-1154.

11 Combs RM, McAllister M, Payne K et al: Understanding the impact of genetic testing for inherited retinal dystrophy. Eur J Hum Genet 2013; 21: 1209-1213.

12 Douglas HA, Hamilton RJ, Grubs RE: The effect of BRCA gene testing on family relationships: a thematic analysis of qualitative interviews. J Genet Couns 2009; 18: $418-435$.
13 Kasparian NA, Meiser B, Butow PN et al: Better the devil you know? High-risk individuals' anticipated psychological responses to genetic testing for melanoma susceptibility. J Genet Couns 2006; 15: 433-447.

14 Graves KD, Peshkin BN, Luta G et al: Interest in genetic testing for modest changes in breast cancer risk: implications for SNP testing. Public Health Genomics 2011; 14: 178-189.

15 Caulfield T, McGuire A: Direct-to-consumer genetic testing: perceptions, problems, and policy responses. Annu Rev Med 2012; 63: 23-33.

16 Lin P-J, Cangelosi MJ, Lee DW et al: Willingness to pay for diagnostic technologies: a review of the contingent valuation literature. Value Health 2013; $1-9$.

17 McKibbin M, Ahmed M, Allsop $M$ et al: Current understanding of genetics and genetic testing and information needs and preferences of adults with inherited retinal disease. Eur J Hum Genet 2014; 22: 1058-1062.

18 Cookson R: Willingness to pay methods in health care: a sceptical view. Health Econ 2003; 12: 891-894.

19 Grosse SD, Wordsworth S, Payne K: Economic methods for valuing the outcomes of genetic testing: beyond cost-effectiveness analysis. Genet Med 2008; 10: 648-654.

20 Blacharski PA: Fundus flavimaculatus; in Newsome DA (ed) Retinal Dystrophies and Degenerations. New York: Raven Press, 1998; pp 135-159.

21 Boughman JA, Conneally PM, Nance WE: Population genetic studies of retinitis pigmentosa. Am J Hum Genet 1980; 32: 223-235. 